CRÍTICA, Revista Hispanoamericana de Filosofía

Vol. XXIX, No. 85 (abril 1997): 65-94

\title{
EL ATOMISMO Y LAS SUSTANCIAS EN DESCARTES
}

Claudia Lorena García

Instituto de Investigaciones Filosóficas

UNAM

La Europa del siglo XVII presencia tanto el nacimiento del cartesianismo como el renacimiento de un atomismo de corte epicúreo. Pierre Gassendi, francés y contemporáneo de Descartes, es el principal promotor del atomismo en Francia. En los círculos intelectuales y científicos de la Gran Bretaña, el atomismo de Gassendi resulta ser enormemente influyente gracias a los esfuerzos proselitistas de filósofos como Charleton y Stanley. ${ }^{1}$ Locke, Newton y muchos otros filósofos naturales de la época llegan a adoptar las tesis fundamentales de este tipo de filosofía corpuscularista; a saber, que el Universo material esta constituido, en última instancia, de por lo menos dos tipos de entidades: (1) un espacio infinito y extenso en tres dimensiones, distinto de la materia, y (2) un gran número de sustancias materiales muy pequeñas, indivisibles y finitas, o átomos. ${ }^{2}$

1 Walter Charleton, Physiologia Epicuro-Gassendi-Charltoniana: or a Fabrick of Science Natural, upon the Hypothesis of Atoms, la. ed., introd. de Robert Hugh Kargon, Londres, 1654, ed. reimpr. Nueva York y Londres, 1966; y Thomas Stanley, The History of Philosophy (3 vols. en 2, Londres, 1655-1660).

2 Una explicación interesante del atomismo de Gassendi, y de su influencia posterior, se encuentra en Lynn Sumida Joy, Gassendi the 
Descartes, por otra parte, rechaza el atomismo por varias razones, entre las cuales la más importante se centra en su convicción de que cualquier partícula de materia, por pequeña que sea, es infinitamente $-o$, como él prefiere expresarlo, indefinidamente- divisible. ${ }^{3}$ Pero, ¿qué es lo que significa esta afirmación para Descartes? ¿Acaso un atomista podría aceptar esta tesis? Aquí exploraré una respuesta a la primera pregunta y defenderé - en contra de ciertos autores - una respuesta negativa a la segunda. En particular, en la sección 1 de este artículo, intentaré mostrar que Descartes tiene una tesis muy fuerte relativa a la divisibilidad indefinida de la materia, una tesis que ningún atomista de la época podría aceptar; a saber, que todo cuerpo finito está actualmente compuesto por un número indefinidamente grande de sustancias corpóreas finitas, realmente distintas entre sí. En las subsecuentes secciones 2 y 3, examinaré con cuidado los conceptos cartesianos de sustancia y de la distinción real entre sustancias, y concluiré que Descartes carece de los recursos teóricos para poder defender su tesis fuerte relativa a la divisibilidad indefinida de la materia; de hecho, concluiré que Descartes no puede afirmar que los cuerpos finitos que constan de partes son sustancias.

\section{Descartes contra los atomistas}

Recientemente, algunos estudiosos de Descartes han sostenido que la tesis cartesiana de la divisibilidad de la materia afirma únicamente que Dios podría partir cualquier pedazo

Atomist: Advocate of History in an Age of Science, Cambridge University Press, Cambridge, 1987, esp. caps. 1 y 9.

${ }^{3}$ Exploraremos la distinción cartesiana entre lo infinito y lo indefinido en la sección 2 del presente ensayo. Nótese también que otra de las razones que Descartes formula en contra del atomista es que la noción de un espacio que podría estar vacío contiene contradicciones. Véase AT VIII-1 49, AT II 482, AT IV 329 y AT V 223-224. 
de materia en un número indefinidamente grande de partículas más pequeñas — algo que un atomista como Gassendi podría aceptar sin ningún problema. Daniel Garber, por ejemplo, sostiene que

Gassendi estaría totalmente dispuesto a aceptar que Dios podría partir un átomo si quisiese. Pero esto es enteramente consistente con el hecho de que Dios creó una cosa que no puede dividirse por ningún medio natural [...] Esto es lo que Descartes debe de establecer para refutar al atomismo, que no existen cuerpos naturalmente indivisibles; una divisibilidad supernatural es, en cierta forma, irrelevante. ${ }^{4}$

En contra de esto, en esta sección argüiré que existe un desacuerdo sustancial entre Descartes y Gassendi en lo que respecta a la divisibilidad de la materia. Defenderé que, aunque es cierto que uno de los argumentos de Descartes a favor de la divisibilidad indefinida de la materia hace referencia al poder de Dios, esta referencia a Dios es sólo una premisa dentro de un argumento más grande cuya conclusión final es que cualquier pedazo de materia está actualmente compuesto de al menos dos sustancias corpóreas finitas (o cuerpos) $)^{5}$ —algo que ningún atomista de ninguna estirpe podría aceptar.

De hecho, pienso que el núcleo del desacuerdo entre Descartes y el atomista reside en la manera como interpretan las implicaciones que tiene el aceptar que Dios podría dividir cualquier pedazo de materia: para el atomista esta afirmación implica solamente que Dios podría generar dos sustancias corpóreas distintas al dividir un átomo; en otras palabras, que no hay contradicción alguna en suponer que un átomo estuviese compuesto de otras dos sustancias. Para

4 Daniel Garber, Descartes' Metaphysical Physics, The University of Chicago Press, Chicago y Londres, 1992, p. 125.

5 Véase AT III 477-478, AT VII 227 y AT VIII-1 28-29. 
Descartes, por otra parte, afirmar que Dios podría separar dos de las partes concebibles de una cosa - por ejemplo, partir un pedazo de materia en dos, o separar una mente de un cuerpo- implica que estas partes son actualmente dos sustancias realmente distintas. Podemos apreciar que esto es lo que la divisibilidad indefinida de la materia significa para Descartes, si notamos que el argumento a favor de tal divisibilidad con frecuencia aparece junto al argumento de la distinción real entre la mente y el cuerpo —un argumento que, como todos sabemos, intenta mostrar que la mente y el cuerpo son dos sustancias numéricamente distintas. Por ejemplo, en la carta a Gibieuf del 19 de enero de 1642, Descartes dice:

La existencia de los átomos, o partes de materia que tienen extensión y que sin embargo son indivisibles, involucra una contradicción, puesto que es imposible tener la idea de una cosa extensa sin al mismo tiempo tener la idea de la mitad o la tercera parte de esa cosa [...] Del simple hecho de que considero las dos mitades de una partícula de materia, por pequeña que sea, como dos sustancias completas cuyas ideas no se tornan inadecuadas por una abstracción de mi intelecto, concluyo con certeza que son realmente divisibles [...] Digo lo mismo acerca del alma y el cuerpo y en general de todas las cosas de las cuales tenemos ideas distintas y completas; a saber, digo que el que sean inseparables involucra una contradicción. (AT III 477-478; las cursivas son mías)

Y, en los Principios de la filosofía I, 60, Descartes afirma explícitamente que "todas y cada una de sus partes [de cualquier sustancia corpórea], como están definidas por nuestro pensamiento (a nobis cogitatione definitam), son realmente distintas de las otras partes de la misma sustancia" (AT VIII-1 28; las cursivas son mías); y añade, "razones similares pueden aducirse a favor de la distinción real entre la mente y el cuerpo". Aquí, pues, se encuentra una afirmación de Descartes que ningún atomista del 
siglo XVII aceptaría: que cualquier partícula de materia, o sustancia corpórea finita, está actualmente compuesta de por lo menos dos sustancias realmente distintas. Esta tesis hace uso del concepto cartesiano de la distinción real; ${ }^{6}$ una distinción que puede hacerse solamente entre sustancias. ${ }^{7}$ Volveré a la cuestión de la distinción real en la sección 3. Pero por lo pronto, me interesa dejar claro que la afirmación de Descartes con respecto a la divisibilidad de la materia es equivalente a afirmar que existe una distinción real (o numérica) entre todas y cada una de las partes concebibles de cualquier cuerpo finito, de las cuales existe un número indefinidamente grande. Examinemos ahora la sustancia en Descartes.

\section{Sustancias y cuerpos}

Existen tres diferentes conceptos cartesianos de sustancia. En esta sección veremos que Descartes tiene problemas teóricos muy serios para poder afirmar que los cuerpos agregativos — es decir, los cuerpos que están compuestos de partes - son sustancias, en cualesquiera de los tres sentidos de 'sustancia' que él maneja. Pero como para Descartes no puede haber cuerpos que no sean agregativos, se sigue que tiene problemas muy serios para poder afirmar que los cuerpos son sustancias $-\mathrm{y}$, por ende, para sostener una posición claramente antiatomista. Veamos.

La primera de las caracterizaciones de la sustancia en Descartes se encuentra en la "Sinopsis" de las Meditaciones:

6 Otros pasajes importantes en los que Descartes habla de la distinción real son: AT VII 169-170, AT VII 221-222 y 227, AT VIII-1 28-29 у AT III 567.

7 Véase AT VIII-1 28: "una distinción real existe sólo entre dos o más sustancias". 
absolutamente todas las sustancias, o cosas que Dios tiene que crear para que existan, son incorruptibles por naturaleza y no pueden dejar de existir a menos que Dios las reduzca a la nada al negarles su concurso. [...] el cuerpo, considerado de manera general, es una sustancia, de forma que éste tampoco perece nunca. (AT VII 14)

De acuerdo con este pasaje, las sustancias creadas son aquellas cosas que (1) sólo dependen del concurso de Dios para poder existir, y (2) sólo Dios puede aniquilarlas. Sin embargo, bajo esta caracterización de 'sustancia', los cuerpos agregativos no serían sustancias puesto que, de acuerdo con Descartes, los cuerpos agregativos pueden perecer fácilmente, no solamente al negarles Dios su concurso. Dice Descartes: "Un cuerpo humano pierde su identidad simplemente como resultado de un cambio en la forma de algunas de sus partes. Y se sigue de esto que el cuerpo puede perecer con mucha facilidad." (AT VII 14) Así, ciertos cambios en un cuerpo - por ejemplo, la pérdida de una de sus partes, por pequeña que sea -8 necesariamente resultan en la pérdida de su identidad.

Debemos enfatizar que, de acuerdo con esta primera caracterización de la sustancia que se encuentra en la "Sinopsis", la materia como un todo no es la única sustancia posible; ${ }^{9}$ los indivisibles del atomista también podrían ser

8 Véase también AT IV 166: "cuando hablamos en general de un cuerpo [particular] nos referimos a una parte determinada de materia, una parte de la cantidad que compone al universo. En este sentido, si se le removiese la más pequeña parte de esa cantidad, inmediatamente juzgaríamos que el cuerpo era más pequeño y que ya no estaba completo; y [además] si se le quitase cualquier partícula de materia, inmediatamente pensaríamos que el cuerpo ya no es [...] numéricamente el mismo" (las cursivas son mías).

9 Hay una razón por la cual Descartes tiene la necesidad de considerar al todo de la materia como una sustancia: él quiere afirmar que Dios es el único ser que podría aniquilar a la materia (al negarle su concurso). Pero para formular esta tesis, Descartes tiene que decir que 
sustancias puesto que, de acuerdo con esta caracterización, una sustancia es aquello que únicamente Dios puede o bien crear o bien destruir; y un átomo, de existir, tendría precisamente estas características. Así que, de acuerdo con la presente noción de sustancia, los átomos podrían ser sustancias genuinas, pero no así los cuerpos agregativos. Quizá ésta es la razón por la cual Descartes más tarde la abandona.

Existe en Descartes una segunda noción de sustancia la cual se encuentra tanto en sus Respuestas a las Objeciones a las meditaciones, como en sus Principios de la filosofía. De acuerdo con esta segunda noción, el término 'sustancia" "no se aplica de manera unívoca a Dios y a sus creaturas". (AT VIII-1 24) A Dios se le llama 'sustancia' en el sentido más propio, ya que Dios no depende de ninguna otra cosa para su existencia. ${ }^{10}$ Por otra parte, algunas de las creaturas de Dios son llamadas 'sustancias' en el sentido de que "necesitan únicamente el concurso de Dios para existir". (AT VIII-1 25) ${ }^{11}$ Esta caracterización de sustancia parece ser la que Descartes finalmente adopta, puesto que aparece repetidamente en diferentes contextos: ${ }^{12}$ una sustancia, nos

la destrucción de un cuerpo particular no implica la aniquilación de su materia. Pero, ¿cómo puede afirmar esto si el cuerpo en cuestión es ese pedazo de materia? Recordemos que Descartes sostiene que si uno le quita una partícula de materia a un cuerpo, el cuerpo resultante ya no es numéricamente idéntico al cuerpo anterior; el cuerpo original ya no existe (véase AT IV 166) - pero, en el proceso de destruir a este cuerpo, no se ha aniquilado ningún pedazo de materia. Por tanto, Descartes necesita poder hablar de la materia como si fuese una cosa en sí misma (es decir, una sustancia) distinta de cualquier cuerpo particular.

${ }^{10}$ Caracterizaciones similares de la sustancia se encuentran también en AT VII 222, en donde las sustancias son aquellas "cosas que subsisten por sí mismas", y en AT VII 226 en donde una sustancia "puede existir por sí misma, esto es, sin la ayuda de ninguna otra sustancia". Véase también AT VII 435.

11 Véase también AT III 429.

12 Véase AT VII 222, AT VII 226 y AT VII 435. 
dice Descartes, es una cosa que subsiste por sí misma, ${ }^{13}$ una cosa que "puede existir en sí, esto es, sin la ayuda de ninguna otra sustancia" (AT VII 226), etc. ${ }^{14}$ La siguiente es una interpretación más precisa de esta segunda noción de sustancia creada en Descartes:

Algo es una sustancia creada si y sólo si (1) es posible que haya un momento en el cual existen solamente Dios y esa otra cosa; y (2) necesariamente, si esa cosa existe en algún momento entonces Dios existe y es la causa de que esa cosa exista en ese momento. ${ }^{15}$

Vemos, entonces, que la pregunta acerca de cómo es que Descartes entiende el concepto de posibilidad (y el de necesidad) es directamente relevante a la cuestión acerca de si él puede afirmar de manera consistente que los cuerpos son sustancias. Por ejemplo, si decir que algo es posible equivale a decir que Dios tiene el poder de crearlo - algo que, en ocasiones, Descartes parece aceptar-, entonces no sólo los cuerpos particulares, sino también sus modos, serían sustancias puesto que, para Descartes, Dios es omnipotente en el sentido amplio de que Dios tiene el poder de crear incluso aquello que involucra una contradicción. ${ }^{16}$ Así, aunque sea contradictorio decir que un modo puede existir en sí — puesto que un modo, por definición, existe

13 Véase AT VII 222.

14 Esta noción de sustancia está relacionada con la tesis de Descartes de que "existen diversos grados de realidad o ser". (AT VII 165)

${ }^{15}$ La interpretación causal de la noción del concurso de Dios es plausible puesto que existe un número de textos en los que Descartes usa un lenguaje causal para describir la acción divina continua requerida para mantener a las cosas finitas en existencia; véase, especialmente, AT VII 109, AT VII 369 y AT III 429.

16 Con respecto a la omnipotencia de Dios en Descartes, véase AT I 149-153 y AT IV 118-119. 
en una sustancia-, ${ }^{17}$ de todas formas Dios podría crear un modo que existiese en sí:

El que yo diga que los modos no son inteligibles aparte de la sustancia en la cual ellos inhieren no debe de entenderse de manera tal que implique que los modos no pueden ser separados de una sustancia por el poder de Dios; puesto que insisto firmemente y creo que Dios puede crear muchas cosas que nosotros somos incapaces de entender. (AT VII 249)

Así, el concepto de posibilidad que Descartes utiliza en su segunda caracterización de la sustancia creada no puede ser entendido en términos del poder ilimitado e ininteligible de Dios, sino en términos de la noción de contradicción, como sigue: algo es posible si no existe ninguna contradicción en suponer que existe o se da. Nos preguntamos, entonces: ¿Es contradictorio suponer que lo único que existe es un cuerpo finito y Dios? Pienso que Descartes no puede dar ninguna respuesta — ni afirmativa ni negativa - a esta pregunta. ¿Por qué?

Si Descartes responde negativamente a la pregunta diciendo que no existe ninguna contradicción en suponer que sólo existe Dios y un cuerpo finito, entonces tendrá que aceptar que un Universo finito es inteligible - algo que él no quiere aceptar. Si, por otra parte, Descartes responde que si existe una contradicción en tal suposición, entonces le será muy difícil afirmar que el universo no es infinitamente, sino únicamente indefinidamente, grande.

Examinemos este dilema. Algo es infinito, de acuerdo con Descartes, si y sólo si existe un argumento correcto que demuestre que aquello no tiene límites. ${ }^{18}$ En este sentido,

17 Véase AT VII 253 y AT VII 434-435.

18 Véase AT V 51-52, en donde Descartes establece de esta manera la diferencia entre lo infinito y lo indefinido. 
nos dice él, sólo Dios es infinito. Por otra parte, una cosa es indefinidamente grande si y sólo si es "imposible probar, o incluso concebir, que tiene límites". (AT V 50) El dilema de Descartes, entonces, es el siguiente:

(1) Si dijese que hay una contradicción en la idea de que sólo Dios y un cuerpo finito existen, entonces los átomos no podrían ser sustancias y, por tanto, no podrían existir - lo cual es una consecuencia deseable para Descartes obtenida, sin embargo, al precio de tener que abandonar la idea de que las sustancias corpóreas finitas de cualquier tipo — divisibles o indivisibles - son posibles. Además, si Descartes aceptase esta alternativa, entonces estaría afirmando una herejía, a saber, que el mundo material es infinito, puesto que si hubiese una contradicción en la idea de que sólo Dios y un cuerpo finito existen, entonces ciertamente existiría un argumento correcto cuya conclusión sería que el mundo material no tiene límites y es, por ende, infinito.

(2) $\mathrm{Si}$, por otra parte, Descartes acepta que es inteligible la idea de que sólo Dios y un cuerpo finito existen, entonces tendría que aceptar la posibilidad de que hay átomos y de que el Universo es finito - dos consecuencias que Descartes no está dispuesto a aceptar. Así, con respecto a la segunda consecuencia, Descartes nos dice que "es imposible [...] concebir que la materia de la cual está hecho el mundo tiene límites". (Ibid.) Y la razón que ofrece es que

[la idea de] que el mundo sea finito o limitado está en conflicto con mi concepto o, lo que es lo mismo, considero que involucra una contradicción, puesto que no puedo sino concebir un espacio más allá de cualesquiera límites que le asignes al mundo. (AT V 345)

Sin embargo, no hay duda de que Descartes dice y sostiene que los cuerpos finitos son sustancias. Hasta ahora he intentado mostrar que la distinción de Descartes entre lo infinito y lo indefinido es incoherente y que, en tanto que 
esto es así, Descartes no tiene ninguna respuesta coherente a dos preguntas centrales relativas a la naturaleza del Universo material; es decir, las preguntas acerca de si los átomos pueden existir y si los cuerpos agregativos pueden ser sustancias — en el segundo sentido de 'sustancia' que hemos distinguido.

Encontramos, sin embargo, un tercer concepto de sustancia, distinto de los anteriores, que Descartes menciona en una de sus cartas a Clerselier (23 de abril de 1649):

Por 'sustancia infinita' entiendo una sustancia que tiene actualmente infinitas e inmensas perfecciones verdaderas y reales. Esto no es un accidente añadido a la noción de sustancia, sino la esencia misma de la sustancia considerada de manera absoluta, sin estar limitada por ningún defecto; estos defectos, con respecto a la sustancia, son accidentes; pero la infinidad o la infinitud no lo es. (AT V 355-356)

Y, en el siguiente párrafo, Descartes añade:

Digo que la noción que tengo del infinito existe en mí antes que la noción de lo finito, puesto que, por el mero hecho de que concibo al ser, o a aquello que es, sin pensar si es finito o infinito, lo que concibo es el ser infinito; pero para poder concebir un ser finito, tengo que quitarle algo a esta noción general de ser, la cual debe por ende de ser anterior. (AT V 356)

Examinemos esta intrigante concepción de sustancia. La sustancia, pura y simple - dice Descartes en esta cartaes aquella entidad cuya idea clara y distinta contiene únicamente lo que, en este pasaje, él llama la 'esencia misma de la sustancia'; es decir, la posesión de perfecciones infinitas e inmensas.

Para entender esta afirmación, debemos notar que, en general, Descartes opone perfecciones a defectos. Las perfecciones son reales; son las propiedades y atributos positivos 
de las cosas. Por el contrario, los defectos, para Descartes - como para cierta tradición escolástica aristotélicacarecen de realidad. Estos escolásticos clasificaban a los defectos como entes de razón, entia rationis, los cuales se dividían en tres clases: negaciones (por ejemplo, la ceguera en un ángel), privaciones o defectos (por ejemplo, la ceguera de Stevie Wonder) e imposibilidades (por ejemplo, un triángulo cuadrado o un humano irracional). ${ }^{19}$ Los entes de razón pueden pensarse — ser objetos en la mente-, pero no existen ni pueden existir puesto que el concepto de un 'ente' de razón (por ejemplo, la ceguera) se opone al concepto de ente, de lo que es real; reales son las sustancias, sus propiedades y capacidades positivas, o perfecciones (por ejemplo, la vista). Por otra parte, a todo aquello que carece de realidad, Descartes lo llama 'no-cosa' (en latín, non res). No-cosas, dice Descartes, son "ciertos seres quiméricos que no pueden existir (êtres chimeriques qui ne peuvent exister)". (AT IX-1 34; las cursivas son mías) ${ }^{20}$

19 Por ejemplo, para Suárez una entidad de razón, o entia rationis, "no tiene en sí misma ninguna realidad (entitatem)". (DM LIV, 1, 6) El concepto de una entidad de razón no tiene nada en común con el concepto de una entidad real, entia realis, por lo cual una entidad de razón no debería, en sentido estricto, llamarse 'entidad' (véase ibid., 1,9). Así, las entidades de razón (al igual que las no-cosas cartesianas) no pueden existir. Un tratamiento conceptual delicado del concepto suareciano de la entidad de razón se encuentra en John P. Doyle, "Suárez on Beings of Reason and Truth (1)", Vivarium, 25, 1987, esp. pp. 53-60 y 69-75.

20 Para Descartes las no-cosas no son reales (AT VII 43, AT IX-1 34), y, entre lo que no es real, Descartes también clasifica a las privaciones, negaciones e imposibilidades (es decir, supuestas entidades cuyos conceptos son autocontradictorios). Véase, por ejemplo, AT VII 190-191: "no se sigue que este defecto [a saber, el error] sea algo real, así como la ceguera no es algo real (realem)"; y AT VII 428: "la forma de la decepción es no-ser (non ens)". Con respecto a las imposibilidades, véase, p.ej., AT VII 138: "cuando habla acerca de un "cuerpo sumamente perfecto' [...] usted está emitiendo una contradicción”. 
Regresemos ahora a la sustancia. En la carta a Clerselier, Descartes nos dice que el concepto de sustancia tout court es idéntico al concepto de sustancia infinita; y que, para poder concebir sustancias finitas, uno tiene que 'quitarle' las nociones de ciertas perfecciones al concepto de sustancia (es decir, al concepto del ser que tiene infinitas perfecciones inmensas). ${ }^{21}$

Esta caracterización de sustancia, sin embargo, parece ser incoherente: si la sustancia es, por definicion, un ser infinitamente perfecto, entonces el concepto de una sustancia finita - de una sustancia defectuosa o imperfectaes autocontradictorio. Y la única manera de evitar esta conclusión, es decir, en efecto, que la palabra sustancia es ambigua puesto que significa, o bien un ser infinitamente perfecto, o bien un ser que tiene algunas, pero no infinitas, perfecciones. ${ }^{22}$

21 Así, un ser humano es mucho más ignorante y débil que Dios; para concebir aquél, uno tiene que concebir primero la sustancia o ser supremo, y después quitarle a ese concepto las ideas de ciertas perfecciones infinitas.

22 Este tercer concepto de sustancia que Descartes formula en la citada carta a Clerselier apela también a otra idea escolástica de acuerdo con la cual existe una jerarquía ontológica universal en la cual cada cosa que existe tiene su lugar, con relación al grado de perfección de esa cosa: Dios es el ser par excellence; sus creaturas son seres pero en menor grado porque son imperfectas; entre más imperfectas son, más alejadas están de Dios y menos se le parecen. Descartes explícitamente adopta una versión de esta idea cuando habla de la existencia de tres grados de perfección o realidad: "Existen diversos grados de realidad o ser (realitatis, sive entitatis); una sustancia tiene más realidad que un accidente o un modo; una sustancia infinita tiene más realidad que una sustancia finita." (AT VII 165) Empero, como ciertos autores contemporáneos han mostrado (véase, p.ej., Eileen O’Neill, “Mind-Body Interaction and Metaphysical Consistency: A Defense of Descartes" en Journal of the History of Philosophy, 2, 1987, pp. 227-245), Descartes hace uso de dos maneras distintas (no obviamente equivalentes) de establecer esta jerarquía ontológica:

(1) a través de la noción de dependencia ontológica: $X$ tiene más 
Así, de acuerdo con este tercer concepto de sustancia, cualquier cosa defectuosa, o carente de perfecciones, sería una sustancia finita. Dejando de lado el problema de distinguir sustancias finitas de modos - los cuales son también cosas imperfectas-, esta tercera caracterización de la sustancia no nos dice nada acerca del tema que nos ocupa aquí — no tiene ninguna implicación acerca de la distinción real entre sustancias finitas: si una sustancia finita es cualquier cosa imperfecta, y dado que la noción misma de ser corpóreo o extenso implica imperfección, ${ }^{23}$ entonces es posible que tanto los átomos como los cuerpos agregativos sean sustancias finitas; de hecho, es posible que el todo de la materia sea también una sustancia finita: todas es-

realidad que $Y$ si y sólo si $Y$ depende para su existencia de $X$, pero $X$ no depende para su existencia de $Y$; o

(2) a través de la noción de perfección, en particular: $X$ tiene más realidad que $Y$ si y sólo si $X$ tiene más perfecciones en un grado más alto que $Y$.

Ahora bien, el concepto de sustancia que aparece en las Respuestas y en los Principios —el segundo antes examinado- está relacionado con la noción de grados de realidad expresada en (1); puesto que, de acuerdo con este concepto, una sustancia finita es aquella cosa que depende de Dios para su existencia (y Dios no depende de ella), pero no depende de ninguna otra cosa. Por otra parte, el (tercer) concepto de sustancia que Descartes formula en la carta a Clerselier se relaciona directamente con el concepto de realidad expresado en (2); de acuerdo con este tercer concepto, una sustancia finita es simplemente aquello que tiene menos perfecciones, o en menor grado, que la sustancia infinita.

Aquellos pasajes en los que Descartes usa 'perfección' y 'realidad' como sinónimos son: AT VII 40, AT VII 161, AT VIII-1 11 y AT I 561. La noción de grados de realidad se encuentra en AT VII 41 y AT VII 165.

23 Véase, por ejemplo, AT VII 138: "Cuando hablas de "un ser corpóreo sumamente perfecto', y entiendes el término 'sumamente perfecto' en su sentido absoluto, de manera tal que por un ser corpóreo se entiende un ser en el cual se encuentran todas las perfecciones, entonces estás emitiendo una contradicción. La naturaleza misma del cuerpo implica muchas imperfecciones." 
tas cosas son imperfectas. Nada acerca de la individuación de las sustancias finitas y de su mutua distinción — nada acerca de la divisibilidad o indivisibilidad de las sustancias corpóreas - se sigue de este concepto de sustancia.

Compárese este tercer concepto con el segundo concepto de sustancia - antes examinado- , de acuerdo con el cual una sustancia finita es aquella que depende únicamente de Dios para existir. De esta caracterización de la sustancia finita naturalmente se desprende una caracterización de la distinción real entre sustancias finitas - que es la que, como veremos, Descartes (en una versión u otra) acepta: una sustancia finita $X$ es realmente distinta de una sustancia finita $Y$ si y sólo si $X$ no depende de $Y$ para su existencia y viceversa; es decir, $X$ podría existir $\sin Y$, y $Y \sin X$. Del tercer concepto cartesiano de sustancia, sin embargo, no se desprende ninguna caracterización de la distinción real - lo cual no es una crítica seria a este tercer concepto, pero sí constituye una razón para no preferirlo por encima del segundo concepto. De hecho, la carta a Clerselier es el único lugar en el que Descartes acepta este - casi paradójico - concepto de sustancia como idéntico a la sustancia infinita; un concepto que, como ya notamos, tiene fuertes tendencias spinozísticas hacia la idea de que sólo Dios puede ser sustancia. ${ }^{24}$

Examinaremos ahora la noción de la distinción real en

24 Debemos notar que, aunque el segundo y el tercer conceptos de sustancia son diferentes, Descartes mismo piensa - la mayoría de las veces, sugiere o supone - que ambos conceptos están relacionados; que tener más y mayores perfecciones es equivalente a gozar de mayor independencia ontológica, y que, entre más perfecto es algo, menor es el número de (las clases de) cosas de las cuales depende para su existencia. En Dios, esto es clarísimo; él es tanto infinitamente perfecto como absolutamente independiente, lo cual no es una coincidencia. No es claro, sin embargo, en virtud de que se da esa equivalencia entre perfección e independencia ontológica, de acuerdo con Descartes. 
Descartes, la cual es crucial al tema que nos ocupa aquí; es decir, al tema de la divisibilidad indefinida de los cuerpos.

3. La distinción real, las sustancias y los átomos

Anteriormente vimos que la estrategia de Descartes en contra de los atomistas consiste en parte en sostener que cualquier partícula de materia es indefinidamente divisible. ${ }^{25}$ Esta afirmación, sin embargo, puede interpretarse de manera tal que el atomista fácilmente estaría de acuerdo con Descartes — si, por ejemplo, la interpretáramos de manera tal que fuese equivalente a afirmar que sólo existe una distinción conceptual entre las partes imaginadas de un pedazo de materia. ${ }^{26}$ Una distinción conceptual (distinctio rationis) - de acuerdo con Descartes y con una larga tradición escolástica que, a este respecto, Descartes siguees una distinción que no tiene ningún fundamento en la realidad y que puede hacerse solamente por medio de una abstracción intelectual. Tal distinción es, en otras palabras, una mera creatura de la mente. La existencia de esta distinción entre $X$ y $Y$ no implica que $X$ y $Y$ sean numéricamente distintos; por el contrario, implica que $X$ y $Y$ son la misma cosa pero considerada bajo diferentes conceptos. El criterio para reconocer la existencia de una distinción conceptual entre $X$ y $Y$, dice Descartes, consiste en que no hay una idea clara y distinta de $X$ que no incluya el concepto de $Y$, y viceversa. ${ }^{27}$

25 Véase AT VIII-1 15 y AT VIII-1 59-60.

${ }^{26}$ La noción de una distinción conceptual en Descartes se encuentra en AT VIII-1 30. Para una explicación clara de las diferentes distinciones en Descartes, véase Norman J. Wells, "Descartes on Distinction", en The Quest for the Absolute, ed. por F.J. Adelmann, Martinus Nijhoff, Boston, 1966, pp. 123-133.

27 Véase AT VIII-1 30. 
Descartes, por tanto, no podría decir que existe solamente una distinción conceptual entre cualesquiera dos partes de una partícula de materia. El atomista, encantado, concordaría con él. De hecho, vimos que la posición de Descartes a este respecto es que existe una distinción real entre esas partes. En las Respuestas al segundo grupo de Objeciones, Descartes nos dice que una distinción real se da entre dos sustancias "cuando cada una de ellas puede existir aparte de la otra". (AT VII 162; las cursivas son mías) Además, en la respuesta a las objeciones de Arnauld, Descartes afirma que "la naturaleza de las sustancias es tal que ellas se excluyen mutuamente unas a otras". (AT VII 227; las cursivas son mías) Y el criterio para reconocer que existe una distinción real entre dos sustancias es el siguiente:

podemos percibir que dos sustancias son realmente distintas del simple hecho de que podemos entender clara y distintamente una aparte de la otra. (AT VIII-1 28)

Más adelante, en este mismo pasaje, Descartes afirma que "todas y cada una de las partes [de cualquier sustancia corpórea], como están definidas por nuestro pensamiento (a nobis cogitatione definitam), son realmente distintas de las otras partes de la misma sustancia". (AT VIII-1 28; las cursivas son mías) Descartes concluirá que toda sustancia corpórea finita está actualmente compuesta de al menos otras dos sustancias corpóreas, a partir de las siguientes premisas: (a) cualquier sustancia corpórea (finita) es tal que podemos concebir clara y distintamente que esa sustancia tiene por lo menos dos partes propias; (b) cada una de las partes de una sustancia corpórea que es concebible clara y distintamente, puede ser concebida clara y distintamente aparte de las otras; (c) $X$ es realmente distinto de $Y$ si $X$ puede ser concebido clara y distintamente aparte de $Y$ y viceversa; (d) si $X$ es realmente distinto de $Y$, entonces $X$ 
y $Y$ son ambas sustancias, y $X$ es numéricamente distinta de $Y$ - en otras palabras, $X$ y $Y$ son dos sustancias distintas. De estas premisas, se sigue que cualquier sustancia corpórea finita está actualmente compuesta de un número indefinidamente grande de sustancias corpóreas finitas y es, en este sentido fuerte, indefinidamente divisible: ¡no hay átomos!

Pero, ¿en qué consiste concebir clara y distintamente una cosa aparte de otra? En el primer grupo de Objeciones a las Meditaciones, Caterus objeta que el criterio que Descartes establece para determinar si hay o no una distinción real entre dos cosas es insuficiente. Por ejemplo, dice Caterus, es posible concebir distintamente la misericordia de Dios aparte de su justicia, sin embargo, nadie pensaría que esos atributos divinos son realmente distintos; Dios es uno y simple..$^{28}$ La respuesta de Descartes en el cuarto grupo de Respuestas consiste en afirmar que, cuando concebimos la misericordia de Dios sin incluir su justicia, estamos concibiendo esos atributos de manera incompleta, haciendo una abstracción por medio del intelecto; y que, "para establecer una distinción real es suficiente que dos cosas puedan ser entendidas como 'completas', y que cada una pueda ser entendida aparte de la otra". (AT VII 221) Esta respuesta no ayuda mucho — como no ayuda tampoco lo que Descartes añade después: una cosa completa es una "sustancia que posee las formas o atributos que me permiten reconocer que es una sustancia". (AT VII 222) ${ }^{29}$ En lo que resta de esta sección, intentaremos clarificar lo que Descartes dice

28 Véase AT VII 100, en donde se encuentra la objeción de Caterus.

29 Conocemos a las sustancias, dice Descartes, sólo al percibir ciertas formas o atributos de esas sustancias, puesto que una sustancia per se, en tanto que es diferente de sus atributos y propiedades, nunca puede ser conocida de manera inmediata. Si tratasemos de quitarle a la sustancia sus atributos, "estaríamos destruyendo todo el conocimiento que tenemos de ella". (AT VII 222) 
en estos pasajes con respecto a la distinción real, y lo que esto implica con respecto a la pregunta acerca de si los cuerpos finitos pueden ser sustancias.

Prima facie, encontramos tres distintas caracterizaciones de la distinción real en Descartes. Primero, está la caracterización del segundo grupo de Respuestas: dos sustancias son realmente distintas cuando cada una de ellas puede existir sin la otra (AT VII 162). De manera más precisa, diremos:

(A) $X$ es realmente distinta de $Y$ si y sólo si es posible que $X$ exista al mismo tiempo que $Y$ no existe, y viceversa.

En segundo lugar, está la noción más vaga de distinción real que se encuentra en la respuesta a Arnauld, y que se entiende en términos de exclusión mutua: "La naturaleza de las sustancias es tal que ellas se excluyen unas a otras." (AT VII 227) Podemos entender esta caracterización de la distinción real de dos maneras distintas:

(B) $X$ es realmente distinta de $Y$ si y sólo si necesariamente, cualesquiera propiedad esencial $P$ es tal que, $X$ tiene $P$ si y sólo si $Y$ no tiene $P$.

Básicamente, una propiedad $P$ es esencial cuando es necesario que cualquier cosa que posee $P$ sea tal que, necesariamente si tal cosa existe entonces posee $P$ - en la terminología de mundos posibles, diríamos que una propiedad esencial es aquella propiedad que, cuando es poseída por una cosa en algún mundo posible, es poseída por esa misma cosa en todos los mundos posibles en los que esa cosa existe. Creo que Descartes estaría de acuerdo en que ser pensante y ser extenso son dos propiedades esenciales en un sentido de 'propiedad esencial' como éste. Entonces, lo que (B) dice es que dos cosas son realmente distintas cuando no pueden compartir ninguna propiedad esencial. Más adelan- 
te examinaremos esta manera de entender la distinción real y concluiremos que es inadecuada. Por lo pronto, veamos una segunda manera de caracterizar de manera precisa esta distinción entendida en términos de la idea de exclusión mutua:

(C) $X$ es realmente distinta de $Y$ si y sólo si necesariamente, cualquier propiedad accidental $P$ es tal que $X$ posee $P$ si y sólo si $Y$ no posee $P$.

Una propiedad accidental es una propiedad que no es esencial. En la terminología cartesiana, (C) afirma que dos cosas realmente distintas no pueden compartir ninguno de sus modos.

Consideremos ahora cada una de las caracterizaciones de la distinción real antes mencionadas. La primera, (A), nos dice que dos sustancias (creadas) son realmente distintas si la existencia de cada una de ellas no implica la existencia de la otra. ${ }^{30} \mathrm{El}$ problema con esta caracterización es que, por sí misma, no constituye un criterio que nos permita decidir qué cosas son realmente distintas de qué otras. ¿Es posible que mi mente exista sin mi cuerpo? ¿Es posible que esta silla exista sin aquella mesa? ¿En que consistiría una justificación a una respuesta afirmativa a estas preguntas? De hecho, vimos que Descartes mismo nos da un criterio para reconocer la existencia de una distinción real entre

30 En términos de la jerarquía ontológica antes mencionada, diríamos que dos sustancias (creadas) son realmente distintas justo cuando tienen el mismo grado de realidad, puesto que son mutuamente independientes. Nótese que, en sentido estricto, no puede decirse que esta caracterización (A) de la distinción real sea general, puesto que no describe correctamente la distinción real que, según Descartes, existe entre la sustancia infinita y las finitas: Dios no es idéntico a ninguna de sus creaturas; sin embargo, dada la primera caracterización (A) de la distinción real, y dado que una sustancia finita no podría existir si Dios no existiese, se seguiría que aquella sustancia no sería realmente distinta de Dios: ¡una conclusión por lo más herética y spinoziana que Descartes jamás aceptaría! 
cualesquiera dos cosas; un criterio distinto de (A), formulado en términos de los atributos o propiedades que dos cosas realmente distintas no pueden compartir. Por ende, debemos ahora examinar las caracterizaciones (B) y (C) de la distinción real antes mencionadas.

Nos preguntaremos, por ende, si Descartes podría establecer una distinción real entre dos cuerpos finitos en términos de los atributos de esos cuerpos — una pregunta que está relacionada con la caracterización (B) de la distinción real- o bien en términos de los modos de esos cuerpos - relacionada con la caracterización (C) de la distinción real. ${ }^{31}$ A fin de cuentas concluiré que Descartes no tiene una manera aceptable de establecer una distinción real entre sustancias finitas, ya sean corpóreas o mentales, en ninguno de los sentidos, (B) o (C), de la distinción real - lo cual, entre otras muchas consecuencias desagradables, lo deja en una posición muy vulnerable frente al atomista.

Pero para apreciar cómo se sigue esta conclusión, necesitamos primero considerar la distinción cartesiana entre modos y atributos. Vimos que una sustancia creada es aquella cosa que necesita únicamente el concurso de Dios para existir. ${ }^{32}$ En contraste con una sustancia finita, están

31 Veremos en esta sección que Descartes caracteriza a los atributos y a los modos de manera distinta, aunque a veces usa 'atributo' de una manera no estricta para referirse tanto a los atributos (en sentido estricto) como a los modos (véase, p.ej., AT VIII-2 348). En otros pasajes, Descartes clasifica a los atributos junto con las propiedades y las cualidades (p.ej., en AT VII 161). También usa 'propiedad' y 'atributo' como si fueran sinónimos en AT VIII-1 25; y 'atributo' para referirse a 'cualquier cosa que debe de inherir en una sustancia para poder existir' (p.ej., en AT VII 222). Aquí nos ceñiremos a la caracterización estricta de atributo de acuerdo con la cual los atributos son distintos de los modos.

32 Aquí daré por hecho que el concepto de sustancia finita que Descartes realmente adopta es el que se encuentra en los Principios y en las Respuestas - es decir, el segundo de los conceptos de sustancia examinados en la sección $1-$ por las razones ahí expuestas. 
aquellas cosas que dependen no únicamente del concurso de Dios, sino también de otras cosas, para poder existir. Los atributos y los modos son de este tipo, puesto que ellos "deben de inherir en algo para poder existir" (AT VII 222), es decir, en alguna sustancia de la cual dependen.

Los modos, sin embargo, son diferentes de los atributos. Descartes caracteriza a los modos a través de la dependencia no-recíproca que tienen con alguna sustancia: un modo no puede existir sin la sustancia de la cual es modo, mientras que la sustancia sí puede existir sin el modo. ${ }^{33}$ En otras palabras, existe una distinción meramente modal entre un modo y la sustancia en la cual éste inhiere. ${ }^{34}$ Por otra parte, la dependencia de un atributo en la sustancia es reciproca: no sólo es el caso que un atributo no puede existir sin la sustancia de la cual es atributo; es también el caso que la sustancia no puede existir sin el atributo. Dice Descartes: los atributos son aquellos "sin los cuales las cosas de las cuales son atributos no podrían existir" (AT IV 348-349) ${ }^{35}$ - en otras palabras, existe una distinción meramente conceptual entre el atributo y la sustancia. Ejemplos de atributos son la existencia y la duración de una sustancia; al igual que la extensión (en general) de una sustancia corpórea, y el pensamiento de una sustancia pensante.

Finalmente estamos en posición de examinar la siguiente caracterización de la distinción real (relacionada con (B)): dos cosas son realmente distintas si y sólo si no compar-

33 Véase, por ejemplo, AT VIII-2 348 y AT IV 348-349.

34 Esto es lo que Norman J. Wells llama una 'separabilidad nomutua'. Véase su "Descartes on Distinction", pp. 116-118. Véase también AT VIII-1 29, AT VIII-2 355 y AT IV 349.

35 Siguiendo a Norman Wells (en su "Descartes on Distinction") podríamos llamar a esto una 'mutua no-separabilidad'. Cuando tratamos de concebir una sustancia finita sin incluir su atributo principal (extensión, en el caso de la sustancia corpórea) la idea resultante es confusa; véase AT VIII-1 31 y AT IX-2 53. 
ten ninguno de sus atributos. Pero esta caracterización es claramente inadecuada puesto que un cuerpo y una mente, ambos, comparten los atributos de existencia y de duración, por lo cual no serían, bajo esta caracterización, realmente distintos. Descartes nunca aceptaría esta consecuencia.

Por lo tanto, debemos ahora explorar la siguiente propuesta: dos cosas son realmente distintas si y sólo si no comparten ningún atributo principal. Un atributo principal de una sustancia, dice Descartes, es

[aquella] propiedad que constituye su naturaleza y esencia, y a la cual se refieren todas sus demás propiedades. Así, la extensión en longitud, anchura y profundidad constituye la naturaleza de la sustancia corpórea; y el pensamiento constituye la naturaleza de la sustancia pensante. (AT VIII-1 25)

Pero si Descartes aceptase que dos cosas son realmente distintas si y sólo si no comparten su atributo principal, entonces tendría que decir que no puede haber dos cuerpos finitos que sean realmente distintos puesto que ningún par de cuerpos finitos difieren en su atributo principal: ambos son cosas extensas - en cuyo caso, solo podría haber una sustancia corpórea. Nótese que obtendríamos la misma consecuencia en lo que respecta a las mentes: Todo par de mentes finitas coinciden en su atributo principal, en cuyo caso podría existir sólo una mente. Otro resultado de corte spinozista claramente inaceptable.

En general, no es posible caracterizar la distinción real en términos de los atributos que dos cosas no comparten: un atributo - ya sea principal o no- es cualquier rasgo general e inmodificable de una sustancia, para Descartes. ${ }^{36} \mathrm{Se}$ sigue que ningún atributo (o conjunto de atributos) podría inducir una distinción real entre dos cosas finitas - ya sean mentes o cuerpos - puesto que, en principio, este atributo

36 Véase AT VIII-1 26. 
podría ser compartido entre dos o más cosas. Por ejemplo, tener forma (en general) cuenta como un atributo, ${ }^{37}$ pero cualesquiera dos cuerpos finitos son idénticos con respecto a esta propiedad.

Exploremos, por tanto, la segunda alternativa; y digamos que Descartes caracteriza la distinción real en términos de los modos, de la siguiente manera: dos cosas son realmente distintas si y sólo si nunca comparten ningún modo. El siguiente pasaje en Descartes indica la plausibilidad de esta manera de entender la distinción real:

la idea de una sustancia con extensión y forma es una idea completa, puesto que puedo concebirla completamente sola, y negar de ella todas las otras cosas de las cuales tengo ideas. (AT III 475)

Pero la respuesta a la pregunta acerca de si esta última caracterización de la distinción real le permite a Descartes afirmar que dos cuerpos finitos son realmente distintos - la respuesta a esta pregunta, repito, depende de qué clase de cosas considera él que son los modos. Más específicamente, si los modos son repetibles, entonces no será una conclusión necesaria que cualesquiera dos cuerpos finitos sean realmente distintos. Veamos.

Intuitivamente diremos que un modo es un repetible sólo si puede inherir ya sea simultánea y/o sucesivamente en más de una sustancia. Para ser más precisos, si un modo es un repetible, entonces es necesario que el modo inhiera en alguna sustancia en todos los momentos en los que existe - pero no necesariamente en la misma sustancia. Por otra parte, si un modo es un irrepetible, entonces es necesario que exista una y sólo una sustancia en la cual el modo inhiere en cada momento en el que existe, y no puede inherir en diferentes sustancias en diferentes momentos.

37 Véase AT VII 223. 
En ciertos pasajes, Descartes acepta explícitamente la tesis de que los modos son irrepetibles. Por ejemplo, en una carta de 1645 o 1646, Descartes nos dice:

La forma y el movimiento son, estrictamente hablando, modos de la sustancia corpórea; porque el mismo cuerpo puede existir en un momento con una forma (hac figura) y en otro con otra (alia), ahora estar en movimiento y luego en reposo; mientras que, por el contrario, ni esta forma (haec figura) ni este movimiento (hic motus) pueden existir sin este cuerpo (hoc corpore). (AT IV 349)

De acuerdo con este pasaje, un modo es un irrepetible que no puede existir sin una cosa particular. ${ }^{38}$ Sin embargo, existen otros pasajes en Descartes que son compatibles con la tesis de que los modos son repetibles. Aquí no dirimiré la cuestión acerca de si los modos son repetibles o irrepetibles para Descartes. Consideraré la pregunta acerca de si dos cuerpos finitos pueden ser realmente distintos — donde 'realmente distintos' se entiende como "no compartir ningún modo' - bajo ambas hipótesis, es decir, bajo las hipótesis de que los modos son repetibles, y de que son irrepetibles, para Descartes.

38 La tesis de que los modos son irrepetibles también está sugerida en los Principios, AT VIII-1 26, en donde parte de la oposición entre atributos y modos se hace en términos de (a) aquello que se considera, de una manera general, como existente en una sustancia (los atributos); y (b) aquello que no se considera de esta manera general (los modos). En otras palabras, entre las cosas que inhieren en las sustancias, se sugiere una oposición entre aquello que es general y aquello que es particular. Hay otros pasajes en los que Descartes considera a los modos como irrepetibles; principalmente AT III 355-356: "Cuando dije que el movimiento es a su determinación como un cuerpo plano es a su superficie [...] estaba comparándolos meramente como uno compararía dos cosas concretas, para mostrar que aquéllos eran diferentes de las cosas que podrían ser consideradas como meras abstracciones" (las cursivas son mías). 
Supongamos, entonces, que los modos son repetibles para Descartes. ${ }^{39} \mathrm{Si}$ esto es así, entonces, un modo debe inherir en al menos una sustancia, pero no necesariamente en la misma sustancia en el mismo o en diferentes momentos. Supongamos también que los cuerpos tienen modos - algo que Descartes claramente acepta. Se sigue que dos cuerpos podrían compartir el mismo modo; por ejemplo, dos cuerpos podrían tener la misma forma o tamaño. Pero entonces no podríamos usar la distinción real - entendida como la exclusión de modos comunes- para afirmar que cualesquiera dos cuerpos son realmente distintos (es decir, son dos sustancias distintas) —ni tampoco podríamos usarla para afirmar que cualquier cuerpo finito es indefinidamente divisible, en el sentido fuerte e interesante de 'divisibilidad' usado por Descartes y mencionado antes.

Consideremos, por tanto, que los modos son irrepetibles para Descartes — después de todo, existe amplia evidencia textual para suponer que esto es así. Vimos que si un modo es un irrepetible, entonces hay una y sólo una sustancia en la cual el modo inhiere en todo momento, y no puede inherir en diferentes sustancias en diferentes momentos. En este sentido, un modo sería, por ejemplo, la forma particular de este cuerpo particular tal que nada sino este cuerpo podría tener esa forma. Ahora bien, si los modos se con-

39 La caracterización de un modo como repetible deja abiertas dos posibilidades distintas pero no mutuamente excluyentes: (1) que un modo inhiera en un momento dado en una sola cosa y después en otra cosa distinta; y (2) que un modo inhiera en más de una cosa al mismo tiempo. La mayoría de las caracterizaciones de un modo (con la posible excepción de la que está en AT VII 434) excluyen esta segunda posibilidad; mientras que la primera posibilidad no se excluye en la mayoría de los pasajes, excepto los que se encuentran en AT IV 349 y AT III 355-356. Sin embargo, debemos tomar en cuenta que Descartes nunca formula la primera posibilidad de manera explícita — nunca afirma explícitamente que un modo pueda existir sucesivamente en dos o más sustancias - lo cual le da cierta plausibilidad a la tesis de que, para Descartes, los modos son irrepetibles. 
ciben de esta manera, y si aceptamos que dos cosas son realmente distintas si y sólo si nunca comparten ninguno de sus modos, entonces se sigue inmediatamente que dos cuerpos son realmente distintos cuando tienen modos distintos.

Pero esta propuesta es insatisfactoria. Concebir los modos como irrepetibles es concebirlos como individuos de cierto tipo. Específicamente, en tanto que irrepetibles, los modos se individuan por medio de las sustancias particulares en las que inhieren. Así, lo que distingue a esta forma cuadrada individual de aquella otra forma cuadrada individual (ambas de idénticas dimensiones) es que aquella forma inhiere en una sustancia $A$ mientras que la otra forma inhiere en una sustancia $B$ realmente distinta de $A$. ¡Pero estábamos tratando de usar los modos para distinguir las sustancias! La moraleja es que uno no puede distinguir los cuerpos finitos por medio de los modos, los cuales a su vez se distinguen por medio de esos mismos cuerpos.

Parecería, entonces, que Descartes no tiene un criterio aceptable para sostener que las mentes y los cuerpos finitos son sustancias. ${ }^{40}$

40 Alguien podría sugerirle a Descartes la siguiente manera natural de establecer un criterio para reconocer la existencia de una distinción real entre dos cuerpos finitos: $X$ es realmente distinto de $Y$ si y sólo si existe un momento en el cual $X$ tiene una propiedad que $Y$ no tiene en ese momento, o viceversa. Esta caracterización de la distinción real tiene varios problemas: (1) que no creo que sea adecuado interpretar de esta manera la idea cartesiana relativa a las sustancias según la cual dos sustancias distintas se excluyen mutuamente (AT VII 227) de manera tal que a una sustancia "puedo concebirla por sí sola, y negar de ella todo lo demás de lo cual tengo una idea" (AT III 475; las cursivas son mías). (2) que esta caracterización sigue teniendo el mismo tipo de problemas que asedian a las otras — a saber, (B) y (C) - puesto que si lo que se afirma es que dos sustancias son realmente distintas cuando existe un momento en el cual no comparten una propiedad esencial, entonces no podríamos afirmar que dos cuerpos particulares son realmente distintos, puesto que en todo momento comparten todas sus propiedades esenciales. Si por otra parte, afirmáramos que dos 


\section{Conclusión}

Del examen hecho en las secciones precedentes, podemos sacar las siguientes conclusiones: Primero, que ninguno de los conceptos de sustancia que están presentes en la obra cartesiana le permiten a Descartes afirmar de manera no problemática que los cuerpos agregativos son sustancias. De acuerdo con el concepto de sustancia presente en la "Sinopsis a las meditaciones", los átomos podrían ser sustancias pero no así los cuerpos agregativos. Además, el concepto de sustancia de los Principios le permitiría a Descartes afirmar que los cuerpos finitos son sustancias sólo si estuviese dispuesto a abandonar la tesis de que podemos demostrar de manera a priori que el Universo no es finito. Por último, el tercer concepto de sustancia, además de prima facie paradójico, no nos ayuda a responder la pregunta acerca de si los cuerpos finitos son sustancias, y por ende, de si son indefinidamente divisibles — es decir, la pregunta acerca de si cada una de las partes de una sustancia corpórea finita es ella misma una sustancia finita realmente distinta de las otras partes.

Estas dificultades, y el hecho de que Descartes pasa de un concepto de sustancia a otro, son síntomas de una tensión teórica presente en su filosofía - una tensión que, como he intentado mostrar, él nunca resuelve y que se debe al hecho de que quiere afirmar, por una parte, que los cuerpos finitos son sustancias y, por otra, que el Universo corpóreo no puede ser finito. En tanto que esta tensión no se resuelva, no existe una respuesta unívoca en Descartes a la pregunta acerca de si los cuerpos finitos - tal y como

sustancias son realmente distintas cuando existe un momento en el que no comparten algún modo, entonces (a) si los modos son repetibles, no sería necesario que dos cuerpos particulares fuesen realmente distintos, y (b) si los modos son irrepetibles, entonces tenemos el mismo problema de circularidad viciosa explicado con anterioridad. 
él los entiende-, y sus partes, son sustancias; por ende, la pregunta de si podría haber átomos.

Segundo, he argumentado los siguientes puntos: (1) que Descartes no puede construir un argumento coherente para sostener que puede haber una multiplicidad de sustancias corpóreas (es decir, dos o más cuerpos finitos realmente distintos entre sí); y (2) que los estándares de racionalidad dictan que Descartes tendría que aceptar, o bien que no hay sustancias finitas, o bien que sólo existen dos sustancias creadas, una extensa y una pensante.

Vemos así, que aun cuando Descartes intenta distinguirse claramente del atomista al afirmar que todo cuerpo finito es una sustancia que está actualmente compuesta de un número indefinidamente grande de sustancias corpóreas finitas, real o numéricamente distintas entre sí —algo que atomistas como Gassendi o Newton ciertamente no aceptarían-, sin embargo no puede defender esta afirmación dadas las maneras como entiende a la sustancia, los modos y la distinción real.

Debemos recalcar, finalmente, que estas reflexiones nos permiten entender cómo y por qué se dan los cambios intelectuales que ocurren de Descartes a Leibniz y a Spinoza; a entender, por una parte, por qué Leibniz niega que los cuerpos cartesianos - es decir, entidades que son agregados de agregados de agregados...ad infinitum - son verdaderas sustancias; y, por otra parte, por qué Spinoza nos dice que sólo hay una sustancia; de hecho, puedo fácilmente imaginarme al filósofo holandés considerando seriamente cómo es que Descartes pudo afirmar que hay una multiplicidad de cuerpos y de mentes. Hemos visto que, por sus propios compromisos teóricos, Descartes tiende hacia conclusiones fuertemente spinozistas. 


\section{SUMMARY}

I argue that Descartes' doctrine concerning the indefinite divisibility of any piece of matter amounts, for him, to the view that any such piece is actually composed of indefinitely many corporeal substances, each of which is really distinct from the others - something that no Seventeenth Century atomist could accept. Then, I distinguish and examine three concepts of substance in Descartes and argue that he cannot unproblematically assert that finite bodies — all of which are aggregates - are substances in either of the three senses of 'substance'. Finally, I examine the different characterizations of the real distinction that, according to Descartes, exists among substances, and argue that he cannot claim that any two finite bodies are really distinct substances; hence, that he cannot support his contention that any piece of matter is indefinitely divisible. I conclude (1) that, ultimately, Descartes cannot sustain a convincing position against the atomist; and (2) that an examination of the notions of substance and of the real distinction in Descartes allow us to understand clearly some of the intellectual shifts that occur from Descartes to Spinoza and Leibniz. 